\title{
Competition between sub-bandgap linear detection and degenerate two-photon absorption in gallium arsenide photodiodes
}

\author{
Benjamin Vest ${ }^{1}$, Baptiste Fix ${ }^{2}$, Julien Jaeck ${ }^{2}$ and Riad Haïdar ${ }^{2}$
}

\begin{abstract}
This letter is on the response of gallium arsenide $p-i-n$ diodes to sub-bandgap photons (1.55 $\mu \mathrm{m})$. We investigate the various regimes of sub-bandgap operation by using different light sources delivering pulses ranging from nanosecond to microsecond durations. We evidence two regimes : a regime of degenerate two-photon absorption, with a clear quadratic dependence with respect to the incident flux, and a sub-bandgap, temperature dependant linear regime, that drives photocurrent generation at lower power densities. Both processes are associated to a very low quantum efficiency, around 10-8. We then determine absorption coefficients as well as trap densities, thanks to a model involving a photo-assisted Shockley Read Hall effect.
\end{abstract}

Keywords: Gallium arsenide, Two-photon absorption, Non-linear optics, Detection, Defects

\section{Background}

Semiconductors materials with high non-linear coefficients, such as GaAs or InP are good materials for numerous optical devices, such as optical switches [1], logic gates [2], and quantum detectors [3]. Two-photon absorption is a third order optical process describing the quasi simultaneous absorption of a pair of photons in a material, with a quadratic dependence relatively to light intensity.

Inside semiconductors, two photon absorption using sub-bandgap photons can be described by a two-step process: those photons of energy $E<E_{g}$ can promote an electron from the valence band to a virtual state (meaning a non stationary state) in the gap. During the lifetime of this state, given by the second Heisenberg principle $\Delta \tau \geq \frac{\hbar}{2\left(E_{g}-E\right)}$, a second photon with enough energy can complete the transition, hence creating an electron-hole pair and generating a photocurrent, named two-photon current (TPC). As the lifetime of this intermediate state is very short in visible gap material (in the fs range), this process is particularly well suited for ultra fast correlation measurements [3-6]. However, the very short lifetime of the virtual state is also responsible for the intrinsically low

*Correspondence: baptiste.fix@onera.fr

2 ONERA, Chemin de la Hunière, 91761 Palaiseau Cedex, France

Full list of author information is available at the end of the article efficiency of this process, as it requires the quasi simultaneous occurrence of two photons in this time interval. As a consequence, common two-photon absorption applications in semi-conductors are, so far, essentially limited to the study of very fast processes, more generally to high peak intensity regimes, only reached by pulsed light sources.

Yet, TPC can also be considered as an interesting solution to detect infrared light in wide gap semiconductor. Those materials are less sensitive to thermally generated carriers and, if associated with TPC, they would open new applications in the field of room-temperature infrared detection. However, such configuration implies the detection of low optical power delivered by CW light sources which signifies lower levels of generated TPC.

The quadratic behavior of TPC with the optical power has already been checked over several decades by many authors in highly crystalline detectors such as GaAs photocathodes and PMTs [7]. It has also been observed in $\mathrm{GaN}$ photodiodes at high peak- power using pico- and femto-second pulsed lasers [8]. In complement to these previous results, our study of sub-band gap absorption in GaAs photodiodes at lower temporal regimes (from nanoto micro-second pulses) reveals a linear detection process, which appears in competition with TPC. 
Such a process might be attributed either to photoassisted tunneling (PAT) or to photo-assisted ShockleyRead-Hall mechanism (PASRH). Indeed, these two mechanisms involve traps in the energy bandgap and are thus dependant to the quality of the detector in terms of defects.

In this Letter, we investigate the detection of subbandgap photons in a series of commercial GaAs pi-n photodiode at $1.55 \mu \mathrm{m}$ at very low flux. Our study evidences the existence of sub-bandgap absorptions composed of two competitive processes : a linear, temperature dependant, sub-bandgap contribution attributed to PASRH and a quadratic two-photon absorption regime. We also investigate the competition between the two processes in various temporal regimes from ns to $\mu s$ allowing to determine the two-photon absorption coefficient and the trap densities in the photodiode material.

\section{Methods}

\section{Competition between two transition processes}

Our first experimental setup aims at investigating the processes responsible for the sub-bandgap response of several Optowell PP85-B1T0N GaAs PIN photodiode. The ball lens covering the detector has been removed, in order to avoid any effects on the transmission. We focus light emitted by a pulsed laser source, a M-Squared Firefly-IR optical parametric oscillator (OPO 1), delivering pulses of duration $\Delta t=10 \mathrm{~ns}$ under a repetition rate of $f=$ $150 \mathrm{kHz}$ (see Table 1). The light is linearly polarised in the direction maximising the TPC by a polariser. The position of the photodiode is controlled with a 3 -axis motorized stage. Hence, it can be precisely placed at the focal point of the focused beam, thanks to a Z-scan technique [9], realized under a mean incident power of $40 \mathrm{~mW}$. At this focal point, an I-P characteristic of the photodiode is acquired with a Keithley 6430 source-meter, applying a bias voltage from $-1 \mathrm{~V}$ to $0 \mathrm{~V}$. The OPO light is split in two beams using a $\mathrm{ZnSe}$ wedge. The weaker one is used as reference, and its intensity is measured with a fast InGaAs detector. We used optical densities to progressively lower the incident flux, thus giving access to a 4-order magnitude dynamic range of optical power. On the I-P characteristic of Fig. 1, the dark current (around 60 pA) has been substracted, so that the graph only shows the evolution of

Table 1 Summary of the different parameters associated to each experiment

\begin{tabular}{llll}
\hline Setup & OPO1 & OPO2 & AOM \\
\hline Pulse duration $\Delta t$ & $10 \mathrm{~ns}$ & $10 \mathrm{~ns}$ & $1 \mu \mathrm{s}$ \\
Repetition rate $\mathrm{f}$ & $150 \mathrm{kHz}$ & $15 \mathrm{kHz}$ & $150 \mathrm{kHz}$ \\
Wavelength & $1.55 \mu \mathrm{m}$ & $1.55 \mu \mathrm{m}$ & $1.55 \mu \mathrm{m}$ \\
Accessible average power $(\mathrm{W})$ & $10^{-7}-10^{-2}$ & $10^{-4}-10^{-1}$ & $10^{-4}-10^{-2}$ \\
\hline
\end{tabular}

the photogenerated current. One can clearly identify two distinct regimes: the quadratic regime above $1 \mathrm{~mW}$ and a linear behaviour below $100 \mu \mathrm{W}$.

Such a linear regime could be explained by two mechanisms, either the PAT or the PASRH. The PAT is based on the curvature of the energy bands and hence depends on the applied bias. However, our experiments did not show such a dependence for biases ranging from $-1 \mathrm{~V}$ to $0 \mathrm{~V}$, and we thus neglect this contribution. On the other hand, as it has been observed and described in silicon [10], PASRH is based on the ionization of traps from deep levels in the bandgap to the conduction band. This generationrecombination process is driven by the thermal agitation, meaning that PASRH is strongly temperature dependent.

So, in order to check the SRH nature of the transition, the photodiode was placed inside a cryostat and was illuminated by a pulsed light at $1.55 \mu \mathrm{m}$ delivered by a A.P.E nanoLevante OPO (OPO 2) with a repetition rate of $15 \mathrm{kHz}$ (see Table 1). As previously, we achieved two I-P characteristics of the diode while measuring the temperature by a PT100 thermo-resistor glued to the photodiode (see Fig. 2). At room-temperature, a clear transition is observed at $20 \mathrm{pA}$ between a linear and a non-linear regime. In comparison with the first experiment (see Fig. 1), we expect a shift of the TPC due to the difference of duty cycles (factor of 10 between OPO1 and OPO2): indeed, the production of charge carriers by two-photon absorption only occurs when the pulse is illuminating the diode, so that TPC is actually dependant of the peak optical power. One can deduce this factor of ten on TPC from the measurements, corresponding to the ratio of the duty cycles.

At $80 \mathrm{~K}$, the I-P characteristic is quadratic over the two decades of measurement. We can notice that the TPC regime is independent of the temperature. But more importantly, no parasitic linear regime is observable at cryogenic temperature due to the freezing of defects and trap levels contribution to this process.

We now modelize the total photocurrent as the sum of both contributions in order to derive their respective parameters. Photocurrent levels are low compared to the incident optical power, so we will consider that the pump is not depleted leading to a simple expression :

$$
\left\langle J_{p h o t}\right\rangle=\left\langle J_{1 p h}\right\rangle+\left\langle J_{2 p h}\right\rangle=K_{1 p h}(T)\left\langle P_{\text {opt }}\right\rangle+K_{2 p h}\left\langle P_{\text {opt }}\right\rangle^{2}
$$

where $J_{1 p h}$ and $J_{2 p h}$ are the respective contributions of PASRH and TPC, $T$ is the temperature, $P_{\text {opt }}$ is the incident optical power and $\langle\cdot\rangle$ represents the integration over one duty cycle. We can now derive $\eta$, the quantum efficiency of the linear process, describing directly the conversion of photons into electrons, and $\beta$, the degenerate two-photon 

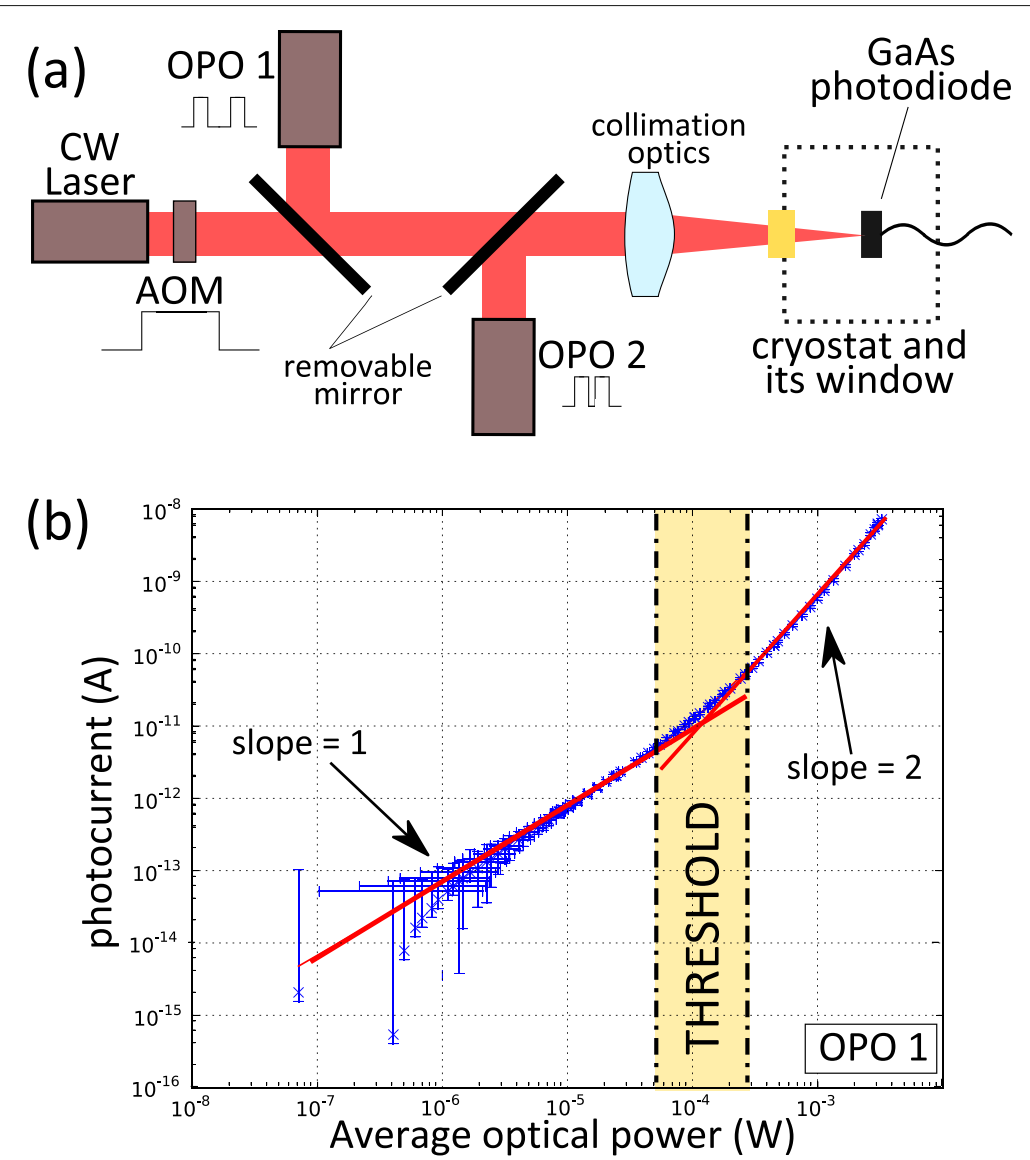

Fig. 1 a Schematic drawing of the experimental set-up. b I-P characteristic of the detector under study at -0.5 V using OPO1 (see Table 1). Two regimes are visible : a linear behavior at low flux regime (slope=1, preponderance of PASRH), a quadratic behavior at higher flux (slope=2, preponderance of TPC)

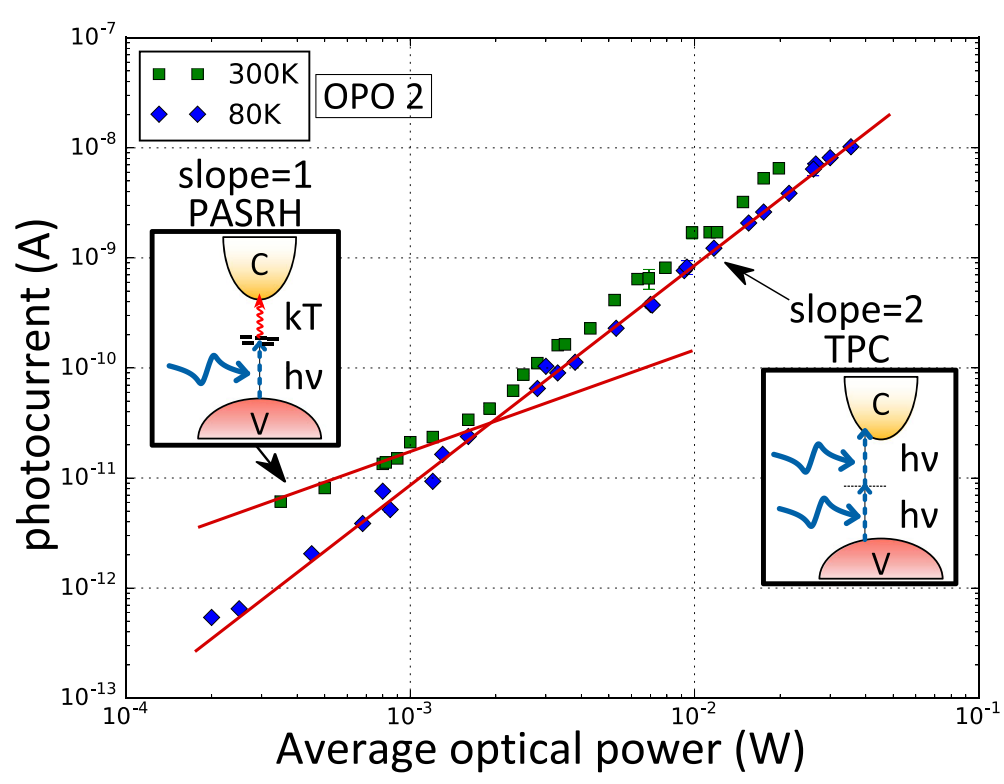

Fig. 2 I-P characteristics of the detector under study mounted in a cryostat using OPO2 (see Table 1). At ambient temperature (green squares), the transition between the linear and the non-linear regime is observed by increasing the incident optical power. At cryogenic temperature ( $80 \mathrm{~K}$ ), there is no linear regime observable due to the freezing of SRH and PASRH processes, thus allowing a pure non-linear detection regime 
absorption coefficient of gallium arsenide at $1.55 \mu \mathrm{m}$ from these coefficients.

$$
\beta=\frac{2 h v}{e} \frac{S}{L} \frac{J_{2 p h}}{P_{o p t}^{2}}
$$

Since our experiment gives access to mean values, we can rewrite this equation as :

$$
\begin{aligned}
& \beta=\frac{2 h v}{e} \frac{S}{L} \frac{\left\langle J_{2 p h}\right\rangle / R_{c}}{\left(\left\langle P_{o p t}\right\rangle / R_{c}\right)^{2}}=\frac{2 h v}{e} \frac{S}{L} K_{2 p h} R_{c} \\
& \eta=\frac{h v}{e} \frac{\left\langle J_{1 p h}\right\rangle}{\left\langle P_{o p t}\right\rangle}=\frac{h v}{e} K_{1 p h}
\end{aligned}
$$

where $e$ is the electron charge, $h v$ the photon energy, $\Delta t$ the pulse duration, $f$ the repetition rate, $R_{c}=\Delta t f$ the duty cycle and assuming a focal spot of area $S=\pi *$ $(40 \mu \mathrm{m})^{2}$ and an active medium thickness of $L=10 \mu \mathrm{m}$ (determined from capacitance-voltage data).

It is well known that beta depends on the polarisation of the light source with respect to the semiconductor crystallographic orientation [11]. Indeed, the two-photon absorption coefficient in GaAs varies with the direction of polarisation as $\sin ^{2}(2 \theta)$ for [100] crystal, as $\left(1+3 \cos ^{2}(\theta)\right) \sin ^{2}(\theta)$ for [110] crystal or with the ellipticity of the polarisation for a [111] crystal. We achieved a set of complementary measurements and found that the GaAs is oriented along its [100] axis in our commercial photodiode, for which $\beta_{[100]}^{\max }=45.4 \pm 7.5 \mathrm{~cm} / \mathrm{GW}$.
On the other hand, estimation of the quantum yield of the linear process is made difficult because of important uncertainties on the photocurrent due to the very weak signal to noise ratio associated to the determination of the incident power at low flux, as well as predominance of TPC at higher fluxes.

\section{Results and discussion}

Improving the measurement precision on the quantum efficiency of the PASRH by changing the temporal regime In order to improve the precision of measurements on the linear process, we change the temporal regime of excitation of the photodiode and we now provide microsecond optical pulses. Indeed, if the two-photon contribution is sensitive to the squared power of the light (meaning that the relevant parameter to quantify photocurrent generation is the squared peak optical power), we emphasize that the linear contribution should be sensitive only to the average number of photons, assuming that the change of temporal regime has no incidence on the defects in terms of response time. By delivering the same number of photons but on a much longer pulse duration, we reduce significantly the non-linear processes as well as we maintain comparable level of linear photocurrent generation (see inset of Fig. 3).

The setup uses now a $1.55 \mu \mathrm{m} \mathrm{CW}$ fiber laser source, and an acousto-optic modulator (AOM). This component chop the continuous wave to define a $1 \mu$ s wide laser pulse, with a similar $150 \mathrm{kHz}$ repetition rate. The light is then

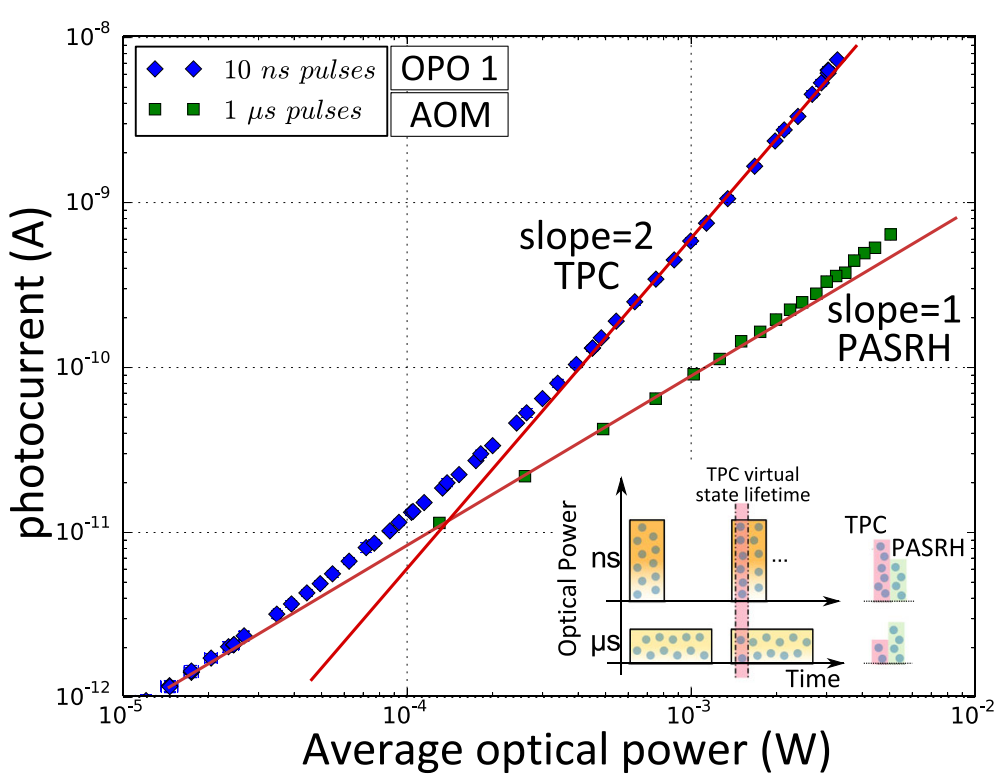

Fig. 3 I-P characteristic of the detector under study in microsecond regime compared with previous results on nanosecond regime (with OPO1) for indentical repetition rate and similar output powers. I-P curve displays a predomining linear behavior, with a smaller quadratic contribution slightly arising. The inset shows the difference in term of photons temporal concentration between the two configuration leading to either a PASRH domination or a TPC domination 
collimated at the output of the fiber and refocused onto the detector (see Table 1).

Figure 3 shows the I-P characteristic obtained with this setup. The preliminary Z-scan alignment step does not display a lorentzian shape anymore, meaning that the non-linear contribution is not predominant. The I-P characteristic is coherent with this first observation, as the linear behaviour of the detector is mainly observed in the intensity range under study. Principles leading the modelization step are nevertheless the same, and we must take into account both contributions. Our modelization leads to values of $\eta=[6.1 \pm 0.6] .10^{-8}$. Now that the incident power is known with a much better precision than previously, the derived value for $\eta$ can be considered as reliable. Yet, due to the microsecond regim, this experiment is not adapted to measure the two-photon absorption coefficient with precision.

The PASRH requires the existence of real electronic states inside the bandgap which are mostly due to defects in the semiconductor [10]. Thus, it is possible to retrieve the density of defects from our experiment. By assuming an optical cross section typically in the $10^{-15} \mathrm{~cm}^{-2}$ range we find a volumic density of defects in the $10^{14} \mathrm{~cm}^{-3}$ range.

Finally, TPC can be compared to PASRH through the introduction of an effective quantum yield. This quantum yield generally describes a probability for a photon to be absorbed in the medium. The quadratic dependance of TPC leads to an intensity-dependent yield, as this probability increases with the number of available photons in the active medium. The inset of Fig. 3 shows a representation of the TPC with respect to the average incident power. It depicts the predominance of absorption of photon pairs in the nanosecond regime, whereas PASRH remains predominant in the microsecond regime. Indeed, the PASRH is proportional to the number of available photons, whereas TPC is sensitive to the photon concentration both in space and time.

\section{Conclusion}

In conclusion, we have observed that a real competition appears in terms of photocurrent generation in our device, limiting possibilities to detect subbandgap photon pairs at low optical powers. However, several strategies are accessible to allow a clear predomination of the TPC signal:

- A significant decrease of the PASRH level, by either cooling the detector [10], or thanks to fewer interactions with trap levels in more controlled active medium and smaller interaction volumes.

- A significant increase of the TPC by enhancing the non-linear process in adequate devices, such as resonant nanostructures [12], quantum wells [13] or photonic crystals [14].

\section{Acknowledgements}

This work is partially supported by a public grant overseen by the French National Research Agency (ANR) as part of the "Investissements d'Avenir" program (Labex NanoSaclay, reference: ANR-10-LABX-0035) and by a DGA-MRIS scholarship. The authors wish to thank Pr. Jacob Khurgin from Johns Hopkins University for fruitful discussions and Pr. Emmanuel Rosencher for his considerable contribution to this work.

\section{Authors' contributions}

$B V$ and $B F$ have led the experiments and have equally contributed to the work JJ has established the experimental protocol. RH has supervised the work. All have contributed to data analysis. All authors read and approved the final manuscript.

\section{Competing interests}

The authors declare that they have no competing interests.

\section{Author details}

${ }^{1}$ Laboratoire Charles Fabry, 2 Avenue Augustin Fresnel, 91127 Palaiseau, France. ${ }^{2}$ ONERA, Chemin de la Hunière, 91761 Palaiseau Cedex, France.

Received: 10 May 2016 Accepted: 21 October 2016

Published online: 13 December 2016

\section{References}

1. Bristow, AD, Rotenberg, N, Van Driel, HM: Two-photon absorption and Kerr coefficients of silicon for 850-2200 nm. Appl. Phys. Lett. 90, 191104-191104 (2007)

2. Liang, T, Nunes, L, Tsuchiya, M, Abedin, K, Miyazaki, T, Van Thourhout, D, Bogaerts, W, Dumon, P, Baets, R, Tsang, H: High speed logic gate using two-photon absorption in silicon waveguides. Opt. Commun. 265, 171-174 (2006)

3. Boitier, F, Dherbecourt, J-B, Godard, A, Rosencher, E: Infrared quantum counting by nondegenerate two photon conductivity in GaAs. Appl. Phys. Lett. 94, 081112 (2009)

4. Lee, $\mathrm{CH}$, Jayaraman, S: Measurement of ultrashort optical pulses by two-photon photoconductivity techniques. Opto-electron. 6, 115-120 (1974)

5. Boitier, F, Godard, A, Rosencher, E, Fabre, C: Measuring photon bunching at ultrashort timescale by two-photon absorption in semiconductors. Nat. Phys. 5, 267-270 (2009)

6. Kikuchi, K: Optical sampling system at $1.5 \mathrm{mu} ; \mathrm{m}$ using two photon absorption in Si avalanche photodiode. Electron. Lett. 34, 1354-1355 (1998)

7. Roth, JM, Murphy, T, Xu, C: Ultrasensitive and high-dynamic-range two-photon absorption in a GaAs photomultiplier tube. Opt. Lett. 27 2076-2078 (2002)

8. Fishman, DA, Cirloganu, CM, Webster, S, Padilha, LA, Monroe, M, David, JH, Van Stryland, EW: Sensitive mid-infrared detection in wide-bandgap semiconductors using extreme non-degenerate two-photon absorption. Nat. Photon. 5, 561-565 (2011)

9. Sheik-Bahae, M, Said, AA, Wei, T-H, Hagan, DJ, Van Stryland, EW: Sensitive measurement of optical nonlinearities using a single beam. Quantum Electron. IEEE J. 26, 760-769 (1990)

10. Vest, B, Lucas, E, Jaeck, J, Haidar, R, Rosencher, E: Silicon sub-bandgap photon linear detection in two-photon experiments: A photo-assisted Shockley-Read-Hall mechanism, Vol. 102 (2013)

11. Dvorak, M, Schroeder, W, Andersen, D, Smirl, A, Wherrett, B: Measurement of the anisotropy of two-photon absorption coefficients in zincblende semiconductors. Quantum Electron. 30, 256-268 (1994)

12. Portier, B, Vest, B, Pardo, F, Péré-Laperne, N, Steveler, E, Jaeck, J, Dupuis, C, Bardou, N, Lemaitre, A, Rosencher, E, et al.: Resonant metallic nanostructure for enhanced two-photon absorption in a thin GaAs pin diode. Appl. Phys. Lett. 105, 011108 (2014)

13. Capasso, F, Sirtori, C, Cho, AY: Coupled quantum well semiconductors with giant electric field tunable nonlinear optical properties in the infrared. Quantum Electron. IEEE J. 30, 1313-1326 (1994)

14. Soljačić, M, Joannopoulos, JD: Enhancement of nonlinear effects using photonic crystals. Nat. Mater. 3, 211-219 (2004) 